Dann unterzog man sie einem FEEL-Test (Facially Expressed Emotion Labeling). Die Ergebnisse im SSRI-Kollektiv waren signifikant schlechter als in der Kontrollgruppe (Harmer CJ et al. Am J Psychiatry 2004).

Agomelatin (Valdoxan ${ }^{\circledR}$ ), das nicht wie SSRI auf die Monoamin-Dysbalance abzielt, sondern auf die zirkadiane Dysregulation, scheint dagegen kein "emotional blunting" zu induzieren. Dafür spricht laut Goodwin das Ergebnis einer weiteren Untersuchung, die mit gleichem Design und Instrumentarium Agomelatin und Placebo verglich und in der sich keine signifikanten
Unterschiede ergaben (Harmer CJ et al. J Psychopharmacol 2010, Epub ahead of print). Außerdem setze bei Agomelatin der antidepressive Effekt früh ein und die Wirksamkeit nehme mit dem Schweregrad der affektiven Störung zu. Das Antidepressivum decke das gesamte Symptomspektrum der Depression ab und weise eine starke anxiolytische Komponente auf.

- Gabriele Blaeser-Kiel

Quelle: Satellitensymposium auf dem European Congress of Neuropsychopharmacology, Paris, September 2011 (Veranstalter: Servier)

\title{
Asthma bronchiale am Arbeitsplatz
}

\section{Patienten wünschen mehr Informationen}

— Knapp zwei Drittel der berufstätigen Asthmatiker sind infolge ihrer Erkrankung in ihrer Leistungsfähigkeit am Arbeitsplatz eingeschränkt, 34\% können ihre Tätigkeit zeitweise nicht ausüben und etwa jeder Vierte hat dadurch berufliche Nachteile. Das hat eine bundesweite, repräsentative Befragung bei 100 Asthmapatienten sowie 100 Ärzten ergeben, die Dr. Dieter Donner, Singen, präsentierte.

Trotz dieser Schwierigkeiten und einem nach eigenen Angaben hohen Informationsbedarf sprechen die Patienten ihre Arbeitsplatzprobleme beim Arzt meist nicht an. Nur 22\% konsultieren den Betriebsarzt. Von den befragten Ärzten erklärte nur rund ein Viertel, von sich aus die

\section{Abbildung 1}

\section{Entwicklung Arbeitsunfähig-} keitstage bei Asthma [Tsd.]
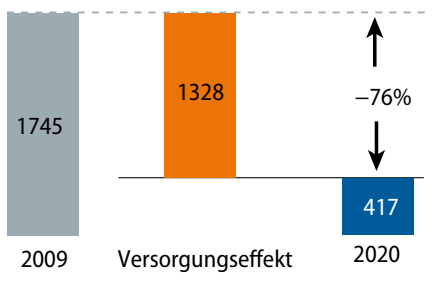

Im Fall einer 100\%igen leitliniengerechten Arzneimittelversorgung könnten die Arbeitsunfähigkeitstage bis 2020 um 1,3 Mio. Tage $(=76 \%)$ reduziert werden.
Thematik zu verbalisieren und Beratung anzubieten.

Defizite gibt es auch in der Behandlung: „Nur jeder zweite Asthmatiker erhält eine leitliniengerechte Behandlung", monierte Dr. Harald Mitfessel, Remscheid. Das zeigt eine Analyse der Daten von mehr als 480000 GKV-Versicherten in Bayern. Es erklärt laut Mitfessel, warum das Therapieziel der Asthmakontrolle trotz umfassender Therapiemöglichkeiten in der Realität oft nicht erreicht wird.

\section{Fixkombination vor der Zulassung}

Eine Verbesserung der Situation erhofft sich der niedergelassene Pneumologe von einer neuen Fixkombination aus Fluticason und Formoterol, die zur Zulassung ansteht. In klinischen Studien, die auf den Nachweis der Non-Inferiority angelegt waren, habe sich das neue Präparat einer etablierten Fixkombination aus LABA und inhalativem Kortikoid als mindestens ebenbürtig erwiesen mit zudem rascher einsetzender Bronchodilatation. Mitfessel: „Wir hoffen, durch die anstehende Erweiterung der Therapiemöglichkeiten bei mehr Patienten als bisher eine gute Asthmakontrolle erzielen zu können."

\section{- Christine Vetter}

Quelle: Pressekonferenz "Unzureichende Asthmakontrolle: Asthma am Arbeitsplatz als unterschätzter Faktor", ERS-Kongress, Amsterdam, September 2011 (Veranstalter: Mundipharma)
Kurz notiert

Erholsamer Schlaf trotz Erkältung

- Zu den Anwendungsgebieten von Klosterfrau Melissengeist zählen Erkältungen und dadurch verursachte Schlafbeschwerden. In einer nicht interventionellen Studie wurden die traditionellen Erfahrungswerte bestätigt. 107 Patienten wurden an 8-9 Tagen mit Klosterfrau Melissengeist behandelt (1-3 x tägl. 5-10

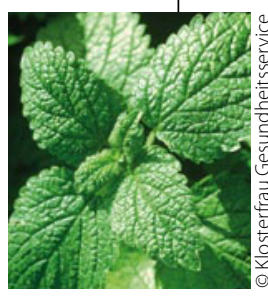

Zitronenmelisse. $\mathrm{ml})$. Bis zum Studienende waren die Schlafbeschwerden bei bis zu $93 \%$ der Patienten verschwunden, die Erkältungssymptome bei bis zu $98 \%$. Die Beschwerden verbesserten sich rasch: nach 3-4 Tagen waren erkältungsbedingte Einschlafstörungen um 65\%, Fließschnupfen, Abgeschlagenheit und Kopfschmerzen um $42 \%$, $38 \%$ bzw. $44 \%$ zurückgegangen.

Klosterfrau

Zeitschrift Schmerztherapie Anfang Dezember erscheint die vierte Ausgabe, u. a. mit folgenden Themen: CME-Beitrag zum Stellenwert der invasiven und interventionellen Schmerztherapie; Therapie von Lymphödemen bei schmerzkranken und palliativen Patienten; Akutschmerzdienst in der Klinik; Diagnostik der Nacken- und Schulterschmerzen; Leitlinien zum Kreuzschmerz (Nationale Versorgungsleitlinie Kreuzschmerz) und LONTS; Schmerztherapie und palliative Versorgung von Parkinsonkranken; Situation in den Altersheimen in puncto Schmerzbehandlung; Rechtmedizinischer Beitrag zur Beweislast bei Behandlungsfehlern und Grundaufklärung vor invasiven Eingriffen. Bezogen werden kann die Zeitschrift über: Deutsche Gesellschaft für Schmerztherapie e.V. - DGS, z. Hd. Frau Krauss, Adenauer Allee 18, D-61440 Oberursel, Tel. 06171/286060.

StK 